\title{
Effect of dry heat, microwave and ultrasonic treatments on physicochemical properties of potato starch with or without pectin
}

\author{
Haiyan Gao ${ }^{1 *}$, Kexin Meng ${ }^{1}$, Jie Zeng ${ }^{1}$, Jikai Jiang ${ }^{1}$, Jinxiang Zhao ${ }^{1,2}$, Meng \\ $\mathrm{Cao}^{1}$, Guanglei $\mathrm{Li}^{1}$, Tongchao $\mathrm{Su}^{1}$ \\ ${ }^{1}$ School of Food Science, Henan Institute of Science and Technology, ${ }^{2}$ Xinxiang Vocational and Technological College, \\ Xinxiang 453003, China
}

*For correspondence: Email: gaohaiyan127@163.com; Tel: +86-13523856903

\begin{abstract}
Purpose: To investigate the effects of dry heat, microwave and ultrasonic treatments on the physicochemical properties of potato starch alone or blended with pectin.

Methods: The physicochemical properties of potato starch gels prepared using microwave, ultrasonic and dry heat treatments were assessed. Pasting properties, gel strength, thermal properties and crystal texture of the potato starch were determined using Rapid Visco analyzer, texture profile analyzer, differential scanning calorimeter and $x$-ray diffractometer.

Results: Dry heat and ultrasonic treatments significantly increased the peak viscosity of the potato starch, and significantly decreased its setback and pasting temperatures $(p<0.05)$. Dry heat treatment significantly increased the hardness, while dry heat and ultrasonic treatments significantly improved retrogradation of the potato starch $(p<0.05)$. Transparency of potato starch paste was significantly increased by the different treatments, except microwave treatment $(p<0.05)$. Potato starch gels blended with pectin and subjected to any of the treatments exhibited significantly increased hardness, when compared with raw potato starch $(p<0.05)$. The retrogradation of the potato starch was significantly improved by the different treatments. Dry heat and ultrasonic treatments significantly decreased the syneresis of potato starch with or without pectin $(p<0.05)$. The three treatments also significantly affected the gelatinization enthalpy of the potato starch with or without pectin, and exerted some effects on the crystallinity of the gels.

Conclusion: The results obtained in this study suggest that differences in physicochemical properties of potato starch gels are due mainly to the degree of damage to starch granules caused by different treatments. The addition of pectin to potato starch gel greatly improves its hardness and retrogradation.
\end{abstract}

Keywords: Potato starch, Pectin, Ultrasonic treatment, Gelatinization enthalpy, Retrogradation

This is an Open Access article that uses a fund-ing model which does not charge readers or their institutions for access and distributed under the terms of the Creative Commons Attribution License (http://creativecommons.org/licenses/by/4.0) and the Budapest Open Access Initiative (http://www.budapestopenaccessinitiative.org/read), which permit unrestricted use, distribution, and reproduction in any medium, provided the original work is properly credited.

Tropical Journal of Pharmaceutical Research is indexed by Science Citation Index (SciSearch), Scopus, International Pharmaceutical Abstract, Chemical Abstracts, Embase, Index Copernicus, EBSCO, African Index Medicus, JournalSeek, Journal Citation Reports/Science Edition, Directory of Open Access Journals (DOAJ), African Journal Online, Bioline International, Open-J-Gate and Pharmacy Abstracts

\section{INTRODUCTION}

Potato starch, a tuber starch accounts for about $6 \%$ of global starch production, and it is ranked third in the world based on its usefulness. Potato starch is characterized by low gelatinization temperature, large particle diameter, high paste viscosity and high paste transparency. Amylose makes up $23.2 \%$ of potato starch, and it is characterized by high swelling force $(19.0 \mathrm{~g} / \mathrm{g})$, 
high solubility (17.5\%) and high paste viscosity [1].

Blends of starch and non-starch polysaccharide hydrocolloids are used as raw or auxiliary materials for food or drug production, because they have different effects on starch gelatinization, viscosity, flow performance and aging properties of binary gels [2,3]. The key factors that control the formation and properties of a binary gel include molecular weight of the components, polydispersity and proportion of amylose in the starch. The effects of molecular weight and polydispersity on thermodynamic compatibility of polysaccharide ligands interacting with each other can be seen from their effects on water activity in solution [4].

The effects of anionic gum solutions (sodium alginate, sodium carboxymethyl cellulose and sodium xanthate) on properties of potato starch under different $\mathrm{pH}$ conditions (6.0 and 8.0) before dry heat treatment have been reported [5]. In one of such studies, the viscosity of potato starch paste was significantly decreased. Xanthan gum has more pronounced influence on viscosity spectrum of binary gels than sodium alginate or carboxymethyl cellulose (CMC). It restrains particle expansion, and enhances shear stability of the paste. The viscosity and shear stability of potato starch is greatly improved using mixed gums of xanthan and alginate. The viscosity of potato starch is also greatly increased by using sodium alginate at $\mathrm{pH} 6$ and xanthan at $\mathrm{pH} 8$ before heat treatment.

In a previous study, the blending of potato starch with ionic gums such as sodium alginate, CMC, and xanthan ( $1 \%$ based on starch solids) followed by dry heat treatment for 0,2 , or $4 \mathrm{~h}$ at $130{ }^{\circ} \mathrm{C}$ significantly reduced the pasting temperature of the starch. Dry heating with xanthan and CMC increases the paste viscosity of potato starch, while sodium alginate decreased it. Extrapolated onset temperature $\left(T_{0}\right)$, conclusion temperature $\left(T_{c}\right)$, and peak temperature $\left(T_{p}\right)$ of potato starch blended with ionic gums are significantly decreased after dry heat treatment. The gel structure of potato starch blended with CMC appears compressed after dry heat treatment. Dry heat treatment improves functional properties of potato starch to some extent [6]. Guar gum increases the peak and final viscosity of potato starch [1]. Rheological properties of blends of potato starch with guar gum, xanthan gum, and k-carrageenan have been described [3]. However, less attention has been paid to potato starch systems blended with pectin. The aim of this study was to investigate the effects of dry heat, microwave and ultrasonic treatments on the physicochemical properties of potato starch alone or blended with pectin.

\section{EXPERIMENTAL}

\section{Materials}

Potato starch was purchased from Beijing Minsong Economic and Trade Co. Ltd. (China); pectin was obtained from Tang Ruisi Food Materials Inc. (USA), while magnetic stirrer was purchased from Tianjin Honor Instrument Co., Ltd. (China). Mechanical pulverizer was a product of Beijing Ever Bright Medical Treatment Instrument Co., Ltd. (China); Rapid Visco Analyzer (RVA-Series 4) was obtained from Newport Scientific Pty., Ltd. (Australia), while Texture Analyzer (SMS, model TA-XT2i) was purchased from Stable Micro System, (England). Boiling water bath (HH-4 digital oscillator) was a product of Changzhou Guohua Electric Co., Ltd. (China); 752N spectrophotometer was purchased from Shanghai Jingke Industrial Co., Ltd. (China), while TDL-40B centrifuge was obtained from Shanghai Anke Scientific Instrument Factory, (China). Differential scanning calorimeter (Q200) was a product of TA instruments Waters LLC, (USA), and X-ray diffractometer was purchased from PAN analytical X'Pert3 Powder, (Netherlands).

\section{Treatments}

\section{Simple blend}

Pectin ( $3 \mathrm{~g}$ ) was dissolved in $170 \mathrm{ml}$ distilled water and stirred vigorously. This was followed by addition of $100 \mathrm{~g}$ of potato starch. The mixture was stirred on a magnetic stirrer for $2 \mathrm{~h}$ at room temperature, and then dried to constant weight in an oven at $40{ }^{\circ} \mathrm{C}$. The blend of potato starch and pectin was crushed using a pulverizer and passed through a $150 \mu \mathrm{m}$ sieve. The raw potato starch without pectin was subjected to the same procedure and used as the control.

\section{Dry heat treatment of potato starch}

The blend of potato starch and pectin was subjected to dry heating at $120^{\circ} \mathrm{C}$ for $3 \mathrm{~h}$ in the oven, and then cooled at room temperature. The control starch without pectin was subjected to the same dry heat treatment and was used for comparison with the blend.

\section{Microwave treatment}

The blend of potato starch and pectin was microwaved with medium heat for $2 \mathrm{~min}$, and then cooled at room temperature. The raw starch 
without pectin was subjected to the same microwave treatment and was used for comparison with the blend.

\section{Ultrasonic treatment}

Pectin $(3 \mathrm{~g}$ ) was dissolved in $170 \mathrm{ml}$ of distilled water and stirred vigorously. This was followed by addition of $100 \mathrm{~g}$ of potato starch. The mixture was stirred on a magnetic stirrer for $2 \mathrm{~h}$ at room temperature. The resultant solution was then treated with ultrasound. The ultrasonic conditions were set as follows: ultrasonic power $(50 \mathrm{~W})$, whole ultrasound duration (10 $\mathrm{min}$ ) at intervals of $25 \mathrm{sec}$ and ultrasound working time of $4 \mathrm{sec}$. The blend was dried to constant weight in an oven at $40{ }^{\circ} \mathrm{C}$, crushed with a pulverizer, and passed through a $150 \mu \mathrm{m}$ sieve. The raw starch without pectin was treated the same way and used for comparison with the blend.

\section{Assessment of pasting properties}

Moisture content of potato starch was adjusted to $14 \%$ according to sample weight. Then, the pasting properties of the starch $(2.0 \mathrm{~g})$ was measured using Rapid Visco analyzer. The peak, trough, breakdown, final, setback and pasting temperatures, and peak time were recorded and compared [7].

\section{Measurement of gel strength}

Potato starch $(2 \mathrm{~g})$ dissolved in $25 \mathrm{ml}$ of distilled water was placed in an aluminum canister, and completely gelatinized based on the RVA measurement. Then, the resultant paste was stored at $4{ }^{\circ} \mathrm{C}$ for $24 \mathrm{~h}$ to form gel. The texture of the gel was analyzed using texture analyzer fitted with a $36 \mathrm{~mm}$ probe (P36R). The test protocol was set up as follows: pre-test speed was 1 $\mathrm{mm} / \mathrm{sec}$, test speed was $0.5 \mathrm{~mm} / \mathrm{sec}$, post-test speed was $1 \mathrm{~mm} / \mathrm{sec}$, compression ratio was 25 $\%$ deformation while force was $5 \mathrm{~g}$. The procedure was performed in triplicate and the mean taken.

\section{Evaluation of retrogradation}

Retrogradation was measured according to the method described by Wang et al [8]. Starch suspension $(10 \mathrm{mg} / \mathrm{ml})$ prepared with distilled water was put in boiling water bath and heated for $30 \mathrm{~min}$. The solution was mechanically stirred during the heating process and then cooled at room temperature. The resultant paste was transferred at room temperature to $100 \mathrm{ml}$ graduated cylinder and the precipitation volume was observed at intervals of $2 \mathrm{~h}$ for $24 \mathrm{~h}$.

\section{Measurement of paste transparency}

The transparency of potato starch paste was measured according to the method described by $\mathrm{Hu}$ et al, with some modifications [9]. Starch suspension (1\%) was prepared in distilled water, heated in boiling water bath for $30 \mathrm{~min}$, and cooled at room temperature. The starch suspension was constantly stirred to prevent precipitation, and distilled water was added intermittently to keep the total volume of the suspension constant. The transmittance of the starch paste was read at $620 \mathrm{~nm}$ and recorded as the starch transparency.

\section{Freeze-thaw stability studies}

The freeze-thaw stability of potato starch with or without pectin was determined based on the method of Wang et al., with some modifications [10]. Starch suspension (3 \%, w/v) was prepared with distilled water and heated in boiling water bath for $30 \mathrm{~min}$ with moderate mechanical agitation, and then cooled at room temperature. Aliquot of the paste $(30 \mathrm{ml})$ was transferred to 50 $\mathrm{ml}$ cold centrifuge tubes, and then thawed and equilibrated at room temperature for $1.5 \mathrm{~h}$.

The thawed tubes were centrifuged at $3000 \mathrm{~g}$ for $15 \mathrm{~min}$, and the volume of water released from the gel after $1,3,5$, and 7 freeze-thaw cycles was measured using a graduated cylinder and expressed as percent of water separated or syneresis.

\section{Differential scanning calorimetry (DSC)}

Thermal properties of the potato starch were determined using a differential scanning calorimeter fitted with a thermal analysis data station. The starch $(0.1 \mathrm{~g})$ was dispersed in 0.2 $\mathrm{ml}$ distilled water and evenly mixed. The mixed sample (8 - $12 \mathrm{mg}$ ) was put on a steel pan and sealed. The scanning temperature ranged from $30-100{ }^{\circ} \mathrm{C}$ at a ramp rate of $10{ }^{\circ} \mathrm{C} / \mathrm{min}$. The curve peaks were analyzed with TA Universal Analysis instrument.

\section{X-ray diffraction (XRD)}

X-ray diffraction analysis of the starch was performed using an X-ray diffractometer [11]. The samples were first ground and sieved using $125 \mu \mathrm{m}$ screen. The operating conditions were: diffraction angle $(2 \theta)$ of $5-80^{\circ}$, emission slit of $1^{\circ}$, anti-scattering slit of $1^{\circ}$, receiving slit of 0.3 $\mathrm{mm}$, step width of $0.02^{\circ}$, and preset time of 0.1 sec. 


\section{Statistical analysis}

Data are expressed as mean $\pm S D$, and the statistical analysis was performed using Microsoft Excel (2007). The different treatment groups were compared using Duncan multiple test range. Values of $p<0.05$ were considered statistically significant.

\section{RESULTS}

\section{Pasting properties}

As shown in Table 1, in the absence of pectin, the peak viscosity of potato starch was significantly increased by dry heat and supersonic treatments $(p<0.05)$. Dry heat and supersonic treatments significantly decreased the setback and pasting temperatures of potato starch, and the peak viscosity was reached at a shorter time $(p<0.05)$. However, microwave treatment had little effect on the gelatinization of potato starch. On addition of $3 \%$ pectin, the pasting properties of potato starch and pectin blend without any treatments were barely changed, relative to raw potato starch $(p>0.05)$.
However, the pasting properties of the blend of potato starch and pectin were greatly affected by dry heat, microwave, and supersonic treatments $(p<0.05)$. The peak viscosity of the blend was significantly reduced $(p<0.05)$. The trough, final viscosity, setback of the potato starch and pectin blend were also significantly decreased by dry heat treatment $(p<0.05)$.

\section{Gel strength}

Raw starch gel had the weakest strength. The different treatments significantly altered the hardness, gumminess and chewiness of the gels $(p<0.05)$, but there were no significant differences in springer and cohesiveness $(p>$ 0.05). In the absence of pectin, dry heat treatment produced potato starch gel with greater hardness, followed by ultrasonic treatment and lastly, microwave treatment. The addition of pectin significantly increased the hardness of the starch gels $(p<0.05)$. In addition, the hardness of starch gel with pectin was further increased by the different treatments. These results are shown in Table 2.

Table 1: Pasting properties of potato starches treated by different methods

\begin{tabular}{cccccccc}
\hline Treatments & $\begin{array}{c}\text { Peak } \\
\text { /cP }\end{array}$ & $\begin{array}{c}\text { Trough } \\
\text { /cP }\end{array}$ & $\begin{array}{c}\text { Breakdow } \\
\text { n /cP }\end{array}$ & $\begin{array}{c}\text { Final } \\
\text { /cP }\end{array}$ & $\begin{array}{c}\text { Setback } \\
\text { /cP }\end{array}$ & $\begin{array}{c}\text { Pasting } \\
\text { temp } \\
\text { / } \mathbf{C}\end{array}$ & $\begin{array}{c}\text { Peak time } \\
\text { /min }\end{array}$ \\
\hline ck & $2706 \pm 11$ & $2166 \pm 12$ & $540 \pm 10$ & $2886 \pm 11$ & $720 \pm 8$ & $71.8 \pm 0.2$ & $4.53 \pm 0.12$ \\
A & $6672 \pm 12$ & $2652 \pm 15$ & $4020 \pm 14$ & $3114 \pm 15$ & $462 \pm 12$ & $65.6 \pm 0.1$ & $3.60 \pm 0.10$ \\
B & $2821 \pm 14$ & $2288 \pm 12$ & $533 \pm 16$ & $3027 \pm 14$ & $739 \pm 13$ & $72.75 \pm 0.2$ & $4.67 \pm 0.14$ \\
C & $6166 \pm 13$ & $2479 \pm 10$ & $3687 \pm 15$ & $2891 \pm 17$ & $412 \pm 12$ & $67.9 \pm 0.1$ & $3.73 \pm 0.13$ \\
D & $2688 \pm 10$ & $1923 \pm 8$ & $765 \pm 12$ & $2666 \pm 12$ & $743 \pm 8$ & $71.2 \pm 0.1$ & $4.67 \pm 0.10$ \\
E & $860 \pm 9$ & $495 \pm 11$ & $365 \pm 16$ & $617 \pm 10$ & $122 \pm 15$ & $67.9 \pm 0.2$ & $3.73 \pm 0.09$ \\
F & $1672 \pm 12$ & $1306 \pm 12$ & $366 \pm 10$ & $1969 \pm 8$ & $663 \pm 10$ & $68.65 \pm 0.2$ & $4.27 \pm 0.08$ \\
G & $2604 \pm 12$ & $1916 \pm 10$ & $688 \pm 12$ & $2524 \pm 16$ & $608 \pm 10$ & $71.15 \pm 0.2$ & $4.87 \pm 0.09$ \\
\hline
\end{tabular}

ck: Raw potato starch; A: potato starch subjected to dry heat treatment without pectin; B: potato starch subjected to microwave treatment without pectin; C: potato starch subjected to supersonic treatment without pectin; D: blend of potato starch and pectin without any treatment; $\mathrm{E}$ : blend of potato starch and pectin subjected to dry heat treatment; F: blend of potato starch and pectin subjected to microwave treatment; and G: blend of potato starch and pectin subjected to supersonic treatment.

Table 2: Gel properties of potato starches

\begin{tabular}{lccccc}
\hline Treatment & Hardness $/ \mathbf{g}$ & Springer & Cohesiveness & Gumminess $/ \mathbf{g}$ & Chewiness $/ \mathbf{g}$ \\
\hline ck & $59.50 \pm 2.18$ & $0.99 \pm 0.03$ & $0.86 \pm 0.02$ & $51.07 \pm 1.99$ & $51.01 \pm 2.30$ \\
A & $191.51 \pm 2.09$ & $0.98 \pm 0.02$ & $0.94 \pm 0.05$ & $179.56 \pm 2.04$ & $175.91 \pm 2.14$ \\
B & $139.36 \pm 2.80$ & $0.97 \pm 0.05$ & $0.91 \pm 0.06$ & $127.72 \pm 1.86$ & $124.13 \pm 2.54$ \\
C & $165.85 \pm 2.22$ & $0.95 \pm 0.05$ & $0.92 \pm 0.02$ & $153.13 \pm 2.01$ & $146.13 \pm 2.58$ \\
D & $208.63 \pm 2.32$ & $0.95 \pm 0.08$ & $0.93 \pm 0.04$ & $194.39 \pm 1.78$ & $183.84 \pm 2.05$ \\
E & $433.38 \pm 3.02$ & $0.92 \pm 0.04$ & $0.92 \pm 0.05$ & $400.16 \pm 1.96$ & $365.96 \pm 2.62$ \\
F & $219.86 \pm 2.88$ & $0.96 \pm 0.02$ & $0.95 \pm 0.05$ & $209.16 \pm 2.08$ & $201.35 \pm 2.03$ \\
G & $299.26 \pm 2.56$ & $0.98 \pm 0.04$ & $0.95 \pm 0.07$ & $283.42 \pm 2.24$ & $278.98 \pm 2.14$ \\
\hline
\end{tabular}

Note: ck, Raw potato starch; A, Potato starch by dry heating treatment without pectin; B, Potato starch by microwave treatment without pectin; $\mathrm{C}$, Potato starch by supersonic treatment without pectin; $\mathrm{D}$, Blends of potato starch and pectin, without any treatment; $E$, Blends of potato starch and pectin by dry heating treatment; $F$, Blends of potato starch and pectin by microwave treatment; $G$, Blends of potato starch and pectin by supersonic treatment 


\section{Retrogradation}

As shown in Figure 1, in the absence of pectin, the precipitation volume of raw potato starch was significantly reduced and showed obvious retrogradation $(p<0.05)$. The precipitation volume of potato starch subjected to microwave treatment was also significantly reduced $(p<$ 0.05). However, dry heat and ultrasonic treatments significantly improved potato starch retrogradation $(p<0.05)$. The precipitation volume of potato starch subjected to dry heat and ultrasonic treatments after $24 \mathrm{~h}$ of storage were significantly increased $(p<0.05)$. After the addition of pectin, the retrogradation of potato starch subjected to the different treatments were further improved.

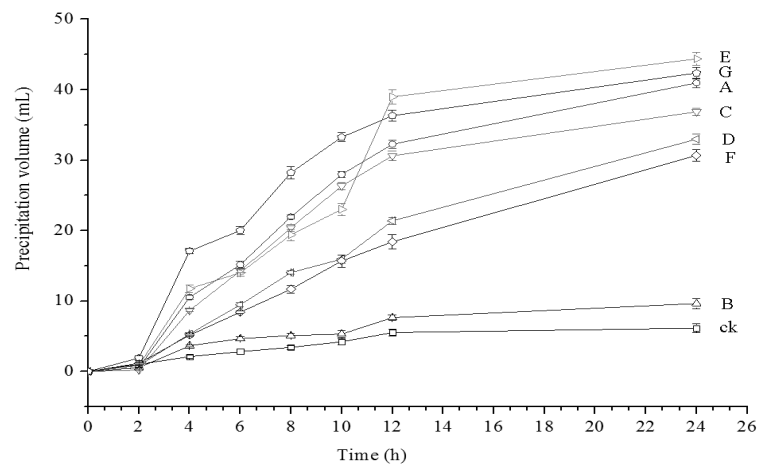

Figure 1: Profiles of retrogradation of potato starch prepared using different methods with or without pectin. ck, Raw potato starch; A, Potato starch by dry heating treatment without pectin; $B$, Potato starch by microwave treatment without pectin; C, Potato starch by supersonic treatment without pectin; D, Blends of potato starch and pectin, without any treatment; E, Blends of potato starch and pectin by dry heating treatment; F, Blends of potato starch and pectin by microwave treatment; $G$, Blends of potato starch and pectin by supersonic treatment

\section{Paste transparency and freeze-thaw stability}

The raw potato starch exhibited low paste transparency $(28 \pm 2 \%)$. The paste transparency of potato starch subjected to the different treatments were significantly increased, except microwave treatment without pectin, when compared with that of raw potato starch $(p<$ 0.05 ). The blend of potato starch and pectin subjected to dry heat treatment showed highest transmittance $(65 \pm 3.5 \%)$. After subjecting the starch gels to different treatments, the photorefractive index and reflectance of the pastes were significantly reduced, and the transparency was significantly improved $(p<$ 0.05 ). Dry heat and ultrasonic treatments significantly reduced the syneresis of potato starch with or without pectin, while microwave treatment significantly increased the water volume separated from the starch, relative to raw potato starch $(p<0.05)$. Thus, freeze-thaw stability of potato starch subjected to dry heating and ultrasonic treatments was significantly increased and exhibited a weak retrogradation trend. These results are shown in Figure 2.

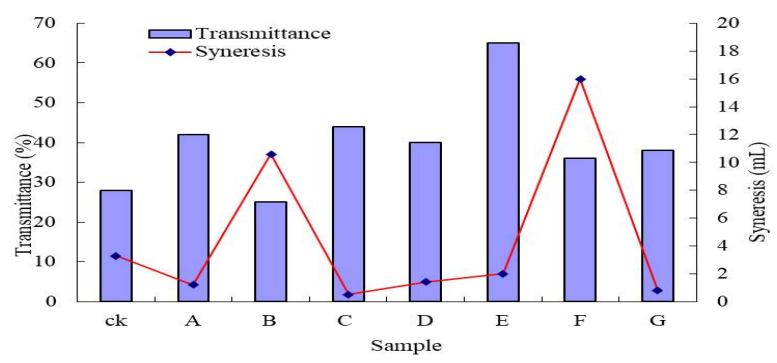

Figure 2: Freeze-thaw stability and paste transparency of potato starch subjected to different treatments with or without pectin. ck, Raw potato starch; A, Potato starch by dry heating treatment without pectin; $B$, Potato starch by microwave treatment without pectin; C, Potato starch by supersonic treatment without pectin; D, Blends of potato starch and pectin, without any treatment; E, Blends of potato starch and pectin by dry heating treatment; $\mathrm{F}$, Blends of potato starch and pectin by microwave treatment; $G$, Blends of potato starch and pectin by supersonic treatment

\section{Thermal characteristics}

Gelatinization temperature for the endothermic transition of the raw potato starch ranged from 62.76 to $83.08{ }^{\circ} \mathrm{C}$, while the enthalpy change $(\Delta \mathrm{H})$ was $4.66 \mathrm{~J} / \mathrm{g}$. The different treatments significantly altered the gelatinization enthalpy of the potato starch with or without pectin $(p<$ $0.05)$. The wider gelatinization temperature $\left(61.47 \pm 0.20\right.$ to $\left.85.35 \pm 0.29^{\circ} \mathrm{C}\right)$ and the lowest gelatinization enthalpy $(2.50 \pm 0.09 \mathrm{~J} / \mathrm{g})$ were found in the blend of potato starch and pectin subjected to dry heat treatment (Table 3 ).

\section{Crystalline properties}

As shown in Figure 3, the raw potato starch powders showed the strongest diffraction peaks at $2 \theta$ angles of $15.143^{\circ}, 17.021^{\circ}$ and $22.956^{\circ}$. However, the first peak $\left(15^{\circ}\right)$ was conspicuously missing from the diffraction patterns of potato starch subjected to different treatments with or without pectin, and the potato starch subjected to microwave treatment without pectin was predominantly associated with B type crystallinity. Although the starch presented a bimodal diffraction distribution, the second peak was weakened and its strength significantly reduced. However, the positions of the two peaks in the derivation patterns were maintained close to 17 and $22^{\circ}$, respectively. 
Table 3: Gelatinization parameters of potato starch subjected to different treatments with or without pectin

\begin{tabular}{ccccc}
\hline Sample & Onset $\left({ }^{\circ} \mathbf{C}\right)$ & Maximum $\left({ }^{\circ} \mathbf{C}\right)$ & Stop $\left({ }^{\circ} \mathbf{C}\right)$ & Area $(\mathbf{J} / \mathbf{g})$ \\
\hline ck & $62.76 \pm 0.42$ & $68.37 \pm 0.33$ & $83.08 \pm 0.31$ & $4.659 \pm 0.15$ \\
A & $63.25 \pm 0.18$ & $69.91 \pm 0.19$ & $85.62 \pm 0.20$ & $2.761 \pm 0.10$ \\
B & $67.26 \pm 0.25$ & $73.89 \pm 0.21$ & $86.57 \pm 0.19$ & $2.723 \pm 0.13$ \\
C & $64.22 \pm 0.22$ & $71.72 \pm 0.18$ & $86.25 \pm 0.25$ & $2.866 \pm 0.12$ \\
D & $64.76 \pm 0.26$ & $72.17 \pm 0.28$ & $84.78 \pm 0.24$ & $2.847 \pm 0.11$ \\
E & $61.47 \pm 0.20$ & $68.49 \pm 0.14$ & $85.35 \pm 0.29$ & $2.504 \pm 0.09$ \\
F & $63.28 \pm 0.21$ & $71.99 \pm 0.26$ & $87.05 \pm 0.20$ & $2.726 \pm 0.12$ \\
G & $65.07 \pm 0.16$ & $72.35 \pm 0.20$ & $85.88 \pm 0.22$ & $2.574 \pm 0.11$ \\
\hline
\end{tabular}

Note: ck, Raw potato starch; A, Potato starch by dry heating treatment without pectin; B, Potato starch by microwave treatment without pectin; $\mathrm{C}$, Potato starch by supersonic treatment without pectin; $\mathrm{D}$, Blends of potato starch and pectin, without any treatment; $E$, Blends of potato starch and pectin by dry heating treatment; $F$, Blends of potato starch and pectin by microwave treatment; G, Blends of potato starch and pectin by supersonic treatment
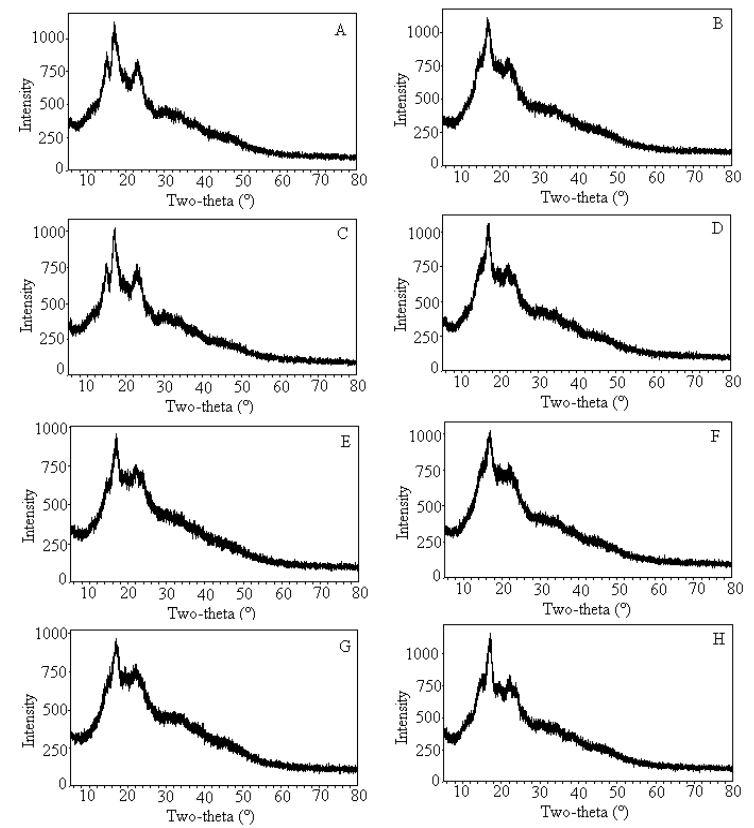

Figure 3: X-ray diffraction patterns of potato starch with or without pectin. A: Raw potato starch; B: potato starch subjected to dry heat treatment without pectin; C: potato starch subjected to microwave treatment without pectin; D: potato starch subjected to ultrasonic treatment without pectin; E: blend of potato starch and pectin, without any treatments; $F$ : blend of potato starch and pectin subjected to dry heat treatment; G: blend of potato starch and pectin subjected to microwave treatment; $\mathrm{H}$ : blend of potato starch and pectin subjected to ultrasonic treatment.

\section{DISCUSSION}

Starch gelatinization is the irreversible destruction of the ordered structure of starch molecules in aqueous solution when the temperature is elevated above the gelatinization temperature under heat treatment [12-14]. In the present study, in the absence of pectin, dry heat and ultrasonic treatments promoted the expansion of starch particles, thereby increasing the peak viscosity. On the other hand, when $3 \%$ pectin was added, the peak viscosities of the starch subjected to dry heat and microwave treatments were significantly decreased, an indication that pectin may hinder the expansion of starch granules. It is likely that the different treatments induced changes in the surface of starch granules, which prevented or delayed the absorption of water molecules. In addition, particle degradation or polymer arrangements may cause starch particles to become more susceptible to shear, resulting in lower peak viscosity [14].

Microwave radiation is a non-ionizing physical modification technique which changes the structural characteristics of potato starch to form microemulsions [15]. Microwave heating rearranges the intramolecular structure of starch, thereby altering its water absorption capacity, solubility, expansion capacity, pasting properties, dehydration, shrinkage and rheological behavior [16-18]. It has been reported that microwave treatment promotes rapid rearrangement of starch molecules at low temperatures [15]. It changes the structure and properties of starch at a lower range of gelatinization temperature. The results obtained in this study suggest that changes in crystalline structures of potato starch with or without pectin when subjected to different treatments may have led to a significant reduction in the amount of energy required for disrupting the $\mathrm{H}$-bonds within junction zones. The structure of the starch molecule may have been damaged during these treatments, or the starch granules may have been encased in a layer of pectin or some reactions occurred with pectin, so that gelatinization of the starch became more difficult.

A highly concentrated starch suspension gelatinizes and cools to form an elastic gel, and gelation depends on time and temperature. Starch gel is a metastable disequilibrium system formed during the aging process of starch and its 
structure changes during storage $[18,19]$. Storage of starch at $4{ }^{\circ} \mathrm{C}$ causes it to age. During storage, a three-dimensional network structure is formed because of the rearrangement of amylose and recrystallization of amylopectin side chains, thereby greatly increasing gel strength [19]. During aging of amylose, 40 - 70 glucose units aggregate to form a double helical structure, while amylopectin is crystallized by rearrangement of its outermost short side chains $(D P=15) \quad[20,21]$. The aggregation and crystallization of amylose is accomplished in the first few hours of storage, while the aggregation and crystallization of amylopectin is completed in the later stage of storage [22].

In this study, the different treatments significantly altered the hardness, gumminess and chewiness of potato starch. The addition of pectin significantly increased the hardness of the starch gels. The results obtained in the present study suggest that hardness, brittleness, elasticity, adhesiveness, degree of conglutination, chewiness and recoverability of starch may be reduced with increased frequency of ultrasound, and are in agreement with those previously reported [9]. The frequency and duration of ultrasound may determine the outcome of ultrasonic treatment. When the water content is sufficient and the heating temperature is elevated above the gelatinization temperature, the starch particles undergo irreversible expansion, leading to leaching of amylose into the solution.

Retrogradation is a reaction that takes place when the amylose and amylopectin chains in cooked, gelatinized starch realign themselves due to hydrogen bond interaction as the cooked starch cools $[8,23]$. Starch retrogradation involves the formation of gel structures, association of amylose chains to form double helix, and the aggregation of amylopectin chains into double helical structures. Generally, amylose retrogrades much faster than amylopectin, and the higher the amylose content, the more rapidly the starch retrogrades [23]. Amylopectin and its intermediates play important roles in retrogradation during refrigeration or storage of starch [24].

The results of this study showed that in the absence of pectin, dry heating and ultrasonic treatments significantly improved retrogradation of potato starch, while microwave treatment had no significant effect on starch retrogradation. However, the addition of pectin significantly improved retrogradation of potato starch regardless of the treatment. The two main factors that affect the transparency of starch paste are size distribution of starch granules and the ratio of amylose to amylopectin [25]. In general, the larger the particle size or the higher the content of amylopectin, the higher the transparency [9]. The paste transparency of potato starch subjected to different treatments were significantly increased, except microwave treatment without pectin, when compared with that of raw potato starch. The highest transmittance was obtained in the blend of potato starch and pectin subjected to dry heat treatment.

"Freeze-thaw" stability is an index of starch retrogradation. The volume of water separated from starch is directly related to the retrogradation trend of the starch [26]. The "freeze-thaw" stability of the whole system becomes worsened when starch retrogradation occurs rapidly [27]. Ice crystals are formed when phase separation occurs due to freezing of starch paste or gels, and during thawing, the paste or gel retains the aqueous phase rich in starch. Increasing the freeze-thaw cycles, increases the degree of phase separation, which is caused by retrogradation of amylopectin. This facilitates the separation of water from dense networks, thereby leading to deterioration of starch quality [24]. Studies have shown that dual-frequency ultrasound exerts some effect on the freeze-thaw stability of corn starch [9].

In this study, dry heat and ultrasonic treatments significantly decreased the syneresis of potato starch with or without pectin, when compared with raw potato starch. These results suggest that dry heat and ultrasonic treatments may improve the freeze-thaw stability of potato starch and lead to a weak retrogradation trend. Thermal enthalpy is a measure of the crystallinity of amylopectin which reflects the quality and quantity of starch crystals, as well as the energy required to disrupt the hydrogen bonds in the junction zones [28,29].

It has been reported that dual frequency ultrasonic treatment could reduce the enthalpy of corn starch, relative to single frequency ultrasound [9]. Dry heat, microwave and ultrasonic treatments significantly affected the gelatinization enthalpy of the potato starch with or without pectin. Wider gelatinization temperature range and the lowest gelatinization enthalpy were found in the blend of potato starch and pectin subjected to dry heat treatment. The raw potato starch powders exhibited the characteristic B-type of crystallinity [30]. It has been reported that ultrasound may not damage the crystal structure of corn starch, and that the peak intensity of the characteristic diffraction 
may be significantly decreased [9]. Studies have also shown that diffused diffraction characteristic is enhanced and the crystal lattice arrangement of corn starch significantly decreased after ultrasound treatment [9].

The results obtained from crystallinity study are not in agreement with those previously reported. This may be due to the differences in raw materials or ultrasonic conditions applied. The first peak $\left(15^{\circ}\right)$ was missing from the diffraction patterns of potato starch subjected to different treatments regardless of whether they were blended with or without pectin. However, the starch subjected to microwave treatment without pectin retained the characteristic $B$ type crystallinity. The first peak of potato starch diffraction pattern was not observed in the presence or absence of pectin, an indication that the treatments may have exerted some effects on the crystal structure of the starch.

\section{CONCLUSION}

The results obtained in this study suggest that differences in the physicochemical properties of potato starch gels are due mainly to the degree of damage to starch granules caused by different treatments. The addition of pectin to potato starch gel greatly improves its hardness and retrogradation, while dry heating treatment enhances the transparency of the starch paste. Moreover, the crystal structure of potato starch and pectin mixture changed following the various treatments.

\section{DECLARATIONS}

\section{Acknowledgement}

This work was financially supported by the Program for Innovative Research Talents (in Science and Technology) of the University of Henan Province (no. 16HASTIT015), the Excellent Youth Foundation of the He'nan Scientific Committee (no. 174100510003). The funders had no role in study design, data collection and analysis, decision to publish, or preparation of the manuscript.

\section{Conflict of interest}

The authors declare that no conflict of interest is associated with this work.

\section{Contribution of authors}

We declare that this work was done by the all of author(s) named in this article and all liabilities pertaining to claims relating to the content of this article will be borne by the authors. Haiyan Gao and Jie Zeng conceived and designed the experiments; Kexin Meng, Tongchao Su, Meng Cao, Meng di Song, and Jikai Jiang conducted the research performed the experiments; Kexin Meng analyzed the data; Hanyan Gao contributed materials and analysis tools; Haiyan Gao and Jie Zeng wrote the paper." Jie Zeng had primary responsibility for final content. All authors read and approved the final manuscript.

\section{Open Access}

This is an Open Access article that uses a funding model which does not charge readers or their institutions for access and distributed under the terms of the Creative Commons Attribution License (http://creativecommons.org/licenses/by/ 4.0) and the Budapest Open Access Initiative (http://www.budapestopenaccessinitiative.org/rea d), which permit unrestricted use, distribution, and reproduction in any medium, provided the original work is properly credited.

\section{REFERENCES}

1. Kaur A, Shevkani K, Singh N, Sharma $P$, Kaur S. Effect of guar gum and xanthan gum on pasting and noodlemaking properties of potato, corn and mung bean starches. J Food Sci Technol 2015; 52: 8113-8121.

2. Nandi $S$, Guha P. Modelling the effect of guar gum on physical, optical, barrier and mechanical properties of potato starch based composite film. Carbohydr Polym 2018; 200:498-507

3. Baranowska HM, Sikora M, Kowalski S, Tomasik $P$. Interactions of potato starch with selected polysaccharide hydrocolloids as measured by low-field NMR. Food Hydrocolloid 2008; 22: 336-345

4. Mazurkiewicz J, Rebilas K, Tomasik $P$. Interactions of dextrans with monosaccharides and sugar alcohols. Food Hydrocolloid 2006; 20: 21-23

5. Lim HS, Bemiller JN, Lim Smil. Effect of dry heating with ionic gums at controlled ph on starch paste viscosity. Cereal Chem 2003; 80: 198-202.

6. Sun QJ, Si FM, Xiong L, LJ Chu. Effect of dry heating with ionic gums on physicochemical properties of starch. Food Chem 2013; 136: 1421-1425.

7. Zeng J, Hu YJ, Gao HY, Sun JL, Ma HJ. Fructooligosaccharides impact on the hydration and retro-gradation of wheat starch and gel. Int J Food Prop 2016; 19: 2682-2692

8. Wang W, Zhou HX, Yang H, Cui M. Effects of salts on the freeze-thaw stability, gel strength and rheological properties of potato starch. J Food Sci Technol 2016; 53:3624-3631.

9. Hu A, Li L, Zheng J, Lu J, Meng X, Liu Y, Rizwan-urRehman. Different-frequency ultrasonic effects on

Trop J Pharm Res, July 2019; 18(7): 1372 
properties and structure of corn starch. J Sci Food Agric 2014, 94:2929-34.

10. Wang L, Xie B, Xiong G, Wu W, Wang J, Qiao Y, Liao L. The effect of freeze-thaw cycles on microstructure and physicochemical properties of four starch gels. Food Hydrocolloid 2013; 31:61-67.

11. Sun JL Li XH, Zeng J, Liu BG, Li GL. Characterization of dextrin prepared by common neutral and thermostable a-amylases. J Food Process Pres 2010; 34:621-631

12. Manley $D$, Pareyt $B$, Delcour JA. Wheat flour and vital wheat gluten as biscuit ingredients. In $D$. Manley (Ed.). Technology of biscuits, crackers and cookies 2011;109133.

13. Ross AS. Starch in foods. In Wrolstad RE (Ed.). Food carbohydrate chemistry 2012; 107-131.

14. Keppler S, Bakalis S, Leadley CE, Sahi SS, Fryer PJ. Evaluation of dry heat treatment of soft wheat flour for the production of high ratio cakes. Food Res Int 2018; 107: 360-370.

15. Fan $D$, Wang $L Y$, Zhang $N$, Xiong $L$, Huang $L L$, Zhao JX, Wang MF, Zhang $H$. Full-time response of starch subjected to microwave heating. Scientific Reports 2017; 7: 3967.

16. Lewandowicz G, Jankowski T, Fornal J. Effect of microwave radiation on physico-chemical properties and structure of cereal starches. Carbohyd Polym 2000; 42 : 193-199.

17. Palav T, Seetharaman K. Impact of microwave heating on the physico-chemical properties of a starch-water model system. Carbohyd Polym 2007; 67: 596-604.

18. Xie $Y, H u X$, Jin Z, Xu X, Chen H. Effect of repeated retrogradation on structural characteristics and in vitro digestibility of waxy potato starch. Food Chem 2014; 163: 219-225.

19. Zhou X, Baik B-K, Wang R, Lim S-T. Retrogradation of waxy and normal corn starch gels by temperature cycling. J Cereal Sci 2010; 51: 57-65.
20. Zhou Z, Robards K, Helliwell S, Blanchard C. Effect of the addition of fatty acids on rice starch properties. Food Res Int 2007; 40: 209-214.

21. Matalanis AM, Campanella OH, Hamaker BR. Storage retrogradation behavior of sorghum, maize and rice starch pastes related to amylopectin fine structure. J Cereal Sci 2009;50:74-81.

22. Sodhi NS, Singh N. Morphological, thermal and rheological properties of starches separated from rice cultivars grown in India. Food Chem 2003; 80: 99-108

23. BeMiller JN, Whistler RL (eds). Starch: chemistry and technology, 3rd edn. Academic Press, New York 2009.

24. Mahmoud Z, Sitohy A, Said SE, Physicochemical properties of different types of starch phosphate monoesters. Starch/Starke 2000; 52: 101-105.

25. Arunyanart $T$, Charoenrein S. Effect of sucrose on the freeze-thaw stability of rice starch gels: correlation with microstructure and freezable water. Carbohydr Polym 2008; 74:514-518.

26. Muadklay J, Charoenrein S. Effects of hydrocolloids and freezing rates on freeze-thaw stability of tapioca starch gels. Food Hydrocolloid 2008; 22:1268-1272.

27. Buckow R, Heinz V, Knorr D. High pressure phase transition kinetics of maize starch. J. Food Eng 2007; 81: 469-475.

28. Lazaridou A, Biliaderis CG, Izydorczyk MS. Molecular size effects on rheological properties of oat-glucans in solution and gels. Food Hydrocolloid 2003; 17: 693-712.

29. Htoon A, Shrestha A, Flanagan B, Lopezrubio A, Bird A, Gilbert $E$. Effects of processing high amylose maize starches under controlled conditions on structural organisation and amylase digestibility. Carbohyd Polym 2009; 75: 236-245.

30. Patel H, Royall PG, Gaisford S, Williams GR, Edwards $\mathrm{CH}$, Warren FJ, Flanagan BM, Ellis PR, Butterworth PJ. Structural and enzyme kinetic studies of retrograded starch: Inhibition of a-amylase and consequences for intestinal digestion of starch. Carbohyd Polym 2017; 164: 154-161. 